\title{
BETA BESSEL DISTRIBUTIONS
}

\author{
ARJUN K. GUPTA AND SARALEES NADARAJAH
}

Received 5 December 2005; Revised 4 May 2006; Accepted 7 May 2006

Three new distributions on the unit interval $[0,1]$ are introduced which generalize the standard beta distribution. These distributions involve the Bessel function. Expression is derived for their shapes, particular cases, and the $n$th moments. Estimation by the method of maximum likelihood and Bayes estimation are discussed. Finally, an application to consumer price indices is illustrated to show that the proposed distributions are better models to economic data than one based on the standard beta distribution.

Copyright (c) 2006 Hindawi Publishing Corporation. All rights reserved.

\section{Introduction}

Beta distributions are very versatile and a variety of uncertainties can be usefully modeled by them. Many of the finite range distributions encountered in practice can be easily transformed into the standard distribution. In reliability and life testing experiments, many times the data are modeled by finite range distributions, see, for example, [2].

A random variable $X$ is said to have the standard beta distribution with parameters $v$ and $\mu$ if its probability density function (pdf) is

$$
f(x)=\frac{x^{\nu-1}(1-x)^{\mu-1}}{B(\nu, \mu)}
$$

for $0<x<1, \nu>0$, and $\mu>0$, where

$$
B(a, b)=\int_{0}^{1} t^{a-1}(1-t)^{b-1} d t
$$

denotes the beta function. Many generalizations of (1.1) involving algebraic, exponential, and hypergeometric functions have been proposed in the literature. Some of these are (see [6, Chapter 25] and [5] for comprehensive accounts) 


\section{Beta Bessel distributions}

(i) the four-parameter generalization given by

$$
f(x)=\frac{1}{(d-c) B(a, b)}\left(\frac{x-c}{d-c}\right)^{a-1}\left(1-\frac{x-c}{d-c}\right)^{b-1}
$$

for $c \leq x \leq d, a>0$, and $b>0$ (see [7, Section 3.2] for a reparameterization of this);

(ii) the McDonald and Richards $[9,13]$ beta distribution given by

$$
f(x)=\frac{p x^{a p-1}\left\{1-(x / q)^{p}\right\}^{b-1}}{q^{a p} B(a, b)}
$$

for $0 \leq x \leq q, a>0, b>0, p>0$, and $q>0$;

(iii) the Libby and Novick [8] beta distribution given by

$$
f(x)=\frac{\lambda^{a} x^{a-1}(1-x)^{b-1}}{B(a, b)\{1-(1-\lambda) x\}^{a+b}}
$$

for $0 \leq x \leq 1, a>0, b>0$, and $\lambda>0$;

(iv) the McDonald and $\mathrm{Xu}[10]$ beta distribution given by

$$
f(x)=\frac{p x^{a p-1}\left\{1-(1-c)(x / q)^{p}\right\}^{b-1}}{q^{a p} B(a, b)\left\{1+c(x / q)^{p}\right\}^{a+b}}
$$

for $0 \leq x^{p} \leq q^{p} /(1-c)$, where $a>0, b>0,0 \leq c \leq 1, p>0$, and $q>0 ;$

(v) the Gauss hypergeometric distribution given by

$$
f(x)=\frac{x^{a-1}(1-x)^{b-1}}{(1+z x)^{\gamma}} B(a, b)_{2} F_{1}(\gamma, a ; a+b ;-z)
$$

for $0<x<1, a>0, b>0$, and $-\infty<\gamma<\infty$ (Armero and Bayarri [1]), where

$$
{ }_{2} F_{1}(a, b ; c ; x)=\sum_{k=0}^{\infty} \frac{(a)_{k}(b)_{k}}{(c)_{k}} \frac{x^{k}}{k !}
$$


denotes the Gauss hypergeometric function, where $(f)_{k}=f(f+1) \cdots(f+$ $k-1)$ denotes the ascending factorial;

(vi) confluent hypergeometric distribution given by

$$
f(x)=\frac{x^{a-1}(1-x)^{b-1} \exp (-\gamma x)}{B(a, b){ }_{1} F_{1}(a ; a+b ;-\gamma)}
$$

for $0<x<1, a>0, b>0$, and $-\infty<\gamma<\infty$ (Gordy [3]), where

$$
{ }_{1} F_{1}(a ; b ; x)=\sum_{k=0}^{\infty} \frac{(a)_{k}}{(b)_{k}} \frac{x^{k}}{k !}
$$

is the confluent hypergeometric function.

In this paper, we introduce the first generalizations of (1.1) involving the Bessel function. We refer to them as the beta Bessel (BB) distributions. We propose three BB distributions in all.

For each of the three BB distributions, we derive various particular cases, an expression for the $n$th moment as well as estimation procedures by the method of maximum likelihood and Bayes method (Sections 2 to 4). We also present an application of the proposed models to consumer price indices (Section 5). The calculations involve several special functions, including the modified Bessel function of the first kind defined by

$$
I_{m}(x)=\frac{x^{m}}{\sqrt{\pi} 2^{m} \Gamma(m+1 / 2)} \int_{-1}^{1}\left(1-t^{2}\right)^{m-1 / 2} \exp (x t) d t
$$

the ${ }_{2} F_{2}$ hypergeometric function defined by

$$
{ }_{2} F_{2}(a, b ; c, d ; x)=\sum_{k=0}^{\infty} \frac{(a)_{k}(b)_{k}}{(c)_{k}(d)_{k}} \frac{x^{k}}{k !},
$$

and the ${ }_{2} F_{3}$ hypergeometric function defined by

$$
{ }_{2} F_{3}(a, b ; c, d, e ; x)=\sum_{k=0}^{\infty} \frac{(a)_{k}(b)_{k}}{(c)_{k}(d)_{k}(e)_{k}} \frac{x^{k}}{k !},
$$

where $(f)_{k}=f(f+1) \cdots(f+k-1)$ denotes the ascending factorial. The properties of the above special functions can be found in $[4,11,12]$. 
4 Beta Bessel distributions

\section{BB distribution I}

The first generalization of (1.1) is given by the pdf

$$
f(x)=C x^{\alpha-1}(1-x)^{\beta-1} I_{\nu}(c x)
$$

for $0<x<1, \nu>0, \alpha>0, \beta>0$, and $c \geq 0$, where $C$ denotes the normalizing constant. Application of [12, equation (2.15.2.1)] shows that one can determine $C$ as

$$
\frac{1}{C}=\frac{c^{\nu} \Gamma(\alpha+\nu) \Gamma(\beta)}{2^{v} \Gamma(\alpha+\beta+\nu) \Gamma(\nu+1)}{ }_{2} F_{3}\left(\frac{\alpha+\nu}{2}, \frac{\alpha+\nu+1}{2} ; \nu+1, \frac{\alpha+\nu+\beta}{2}, \frac{\alpha+\nu+\beta+1}{2} ; \frac{c^{2}}{4}\right) .
$$

The standard beta pdf (1.1) arises as the particular case of (2.1) for $c=0$ and $v=0$. Several other particular cases of (2.1) can be obtained using special properties of $I_{\nu}(\cdot)$. Note that

$$
\begin{gathered}
I_{3 / 2}(x)=\sqrt{\frac{2}{\pi}} \frac{x \cosh (x)-\sinh (x)}{x^{3 / 2}}, \\
I_{5 / 2}(x)=\sqrt{\frac{2}{\pi}} \frac{\left(x^{2}+3\right) \sinh (x)-3 x \cosh (x)}{x^{5 / 2}}, \\
I_{7 / 2}(x)=\sqrt{\frac{2}{\pi}} \frac{x\left(x^{2}+15\right) \cosh (x)-3\left(2 x^{2}+5\right) \sinh (x)}{x^{7 / 2}}, \\
I_{9 / 2}(x)=\sqrt{\frac{2}{\pi}} \frac{\left(x^{4}+45 x^{2}+105\right) \sinh (x)-5 x\left(2 x^{2}+21\right) \cosh (x)}{x^{9 / 2}} .
\end{gathered}
$$

More generally, if $v-1 / 2 \geq 1$ is an integer, then

$$
\begin{aligned}
I_{\nu}(x)= & \sqrt{2} \sqrt{x \pi} \exp \left\{\frac{\pi i}{2}\left(\frac{1}{2}-\nu\right)\right\} \\
\times & {\left[\sinh \left(\frac{\pi x}{2}\left(\frac{1}{2}-\nu\right)-x\right) \times \sum_{k=0}^{[(2|\nu|-1) / 4]} \frac{(|\nu|+2 k-1 / 2) !}{(2 k) !(|\nu|-2 k-1 / 2) !(2 x)^{2 k}}\right.} \\
& \left.+\cosh \left(\frac{\pi x}{2}\left(\frac{1}{2}-\nu\right)-x\right) \sum_{k=0}^{[(2|\nu|-3) / 4]} \frac{(|\nu|+2 k+1 / 2) !(2 x)^{-2 k-1}}{(2 k+1) !(|\nu|-2 k-3 / 2) !}\right] .
\end{aligned}
$$




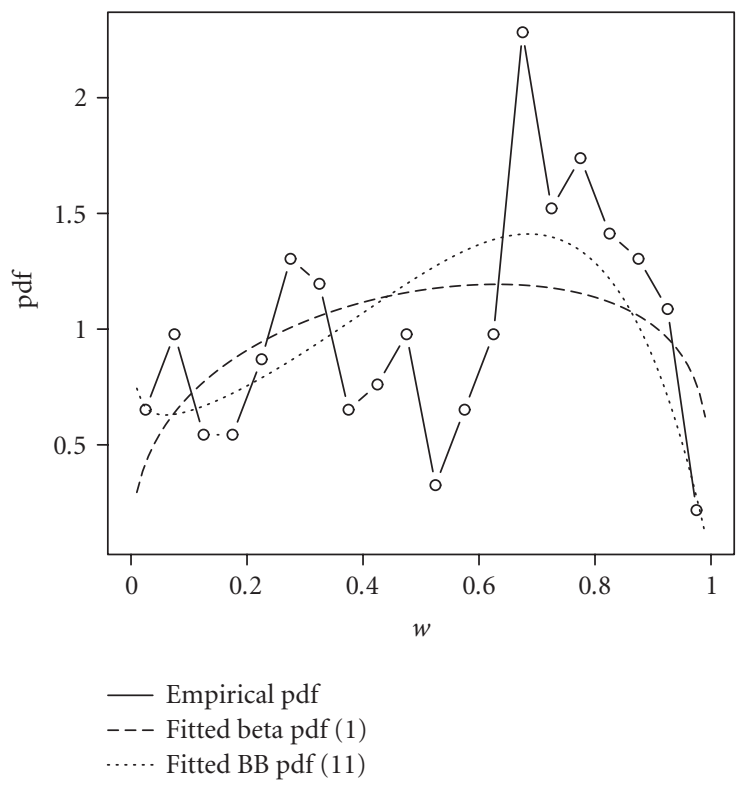

Figure 2.1. The empirical and fitted densities for the consumer price indices of the United States and the United Kingdom $(X=$ consumer price index of the United States and $Y=$ consumer price index of the United Kingdom).

Thus, several particular forms of (2.1) can be obtained for half-integer values of $\nu$. For example, if $\nu=3 / 2$, then (2.1) reduces to

$$
f(x)=C \sqrt{\frac{2}{\pi c^{3}}} x^{\alpha-5 / 2}(1-x)^{\beta-1}\{c x \cosh (c x)-\sinh (c x)\} .
$$

If $v=5 / 2$, then $(2.1)$ reduces to

$$
f(x)=C \sqrt{\frac{2}{\pi c^{5}}} x^{\alpha-7 / 2}(1-x)^{\beta-1} I_{\nu}(c x)\left\{\left(c^{2} x^{2}+3\right) \sinh (c x)-3 c x \cosh (c x)\right\} .
$$

The modes of (2.1) are the solutions of

$$
\frac{\alpha-1}{x}-\frac{\beta-1}{x}+\frac{c I_{\nu-1}(c x)}{I_{\nu}(c x)}=\frac{\nu}{c} .
$$

There could be more than one mode (see Figures 2.1 and 2.2). The $n$th moment of (2.1) can be written as

$$
E\left(X^{n}\right)=C \int_{0}^{1} x^{n+\alpha-1}(1-x)^{\beta-1} I_{\nu}(c x) d x
$$




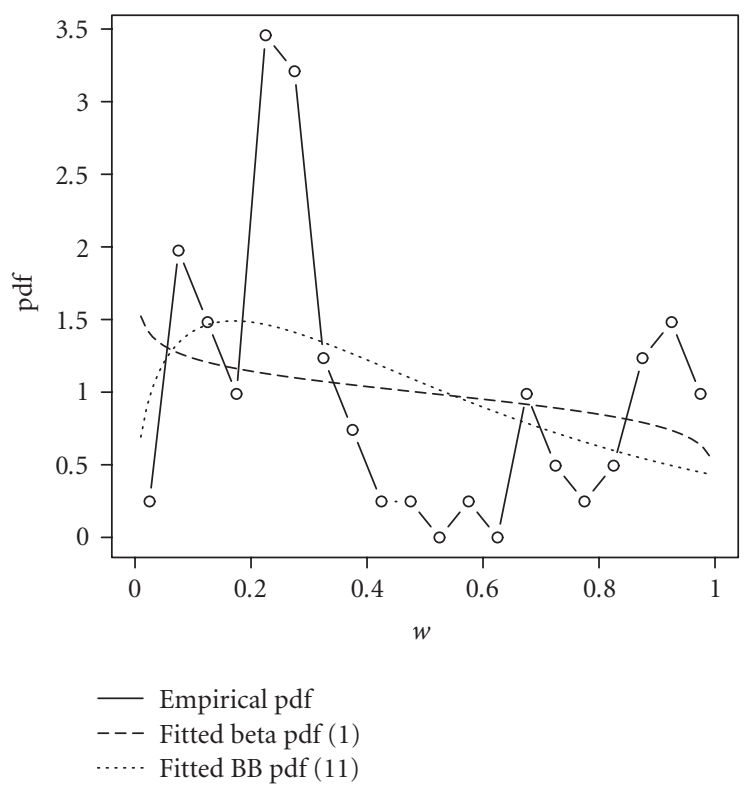

Figure 2.2. The empirical and fitted densities for the consumer price indices of the United States and Germany $(X=$ consumer price index of the United States and $Y=$ consumer price index of Germany).

and an application of [12, equation (2.15.2.1)] shows that (2.8) reduces to

$$
\begin{aligned}
E\left(X^{n}\right)= & \frac{C c^{\nu} \Gamma(n+\alpha+\nu) \Gamma(\beta)}{2^{\nu} \Gamma(n+\alpha+\beta+\nu) \Gamma(\nu+1)} \\
& \times{ }_{2} F_{3}\left(\frac{n+\alpha+\nu}{2}, \frac{n+\alpha+\nu+1}{2} ; \nu+1, \frac{n+\alpha+\nu+\beta}{2}, \frac{n+\alpha+\nu+\beta+1}{2} ; \frac{c^{2}}{4}\right) .
\end{aligned}
$$

For a random sample $w_{1}, \ldots, w_{n}$, the maximum-likelihood estimators (MLEs) of the four parameters in (2.1) are the solutions of

$$
\begin{gathered}
\sum_{i=1}^{n} \operatorname{In} w_{i}=-\frac{n}{C} \frac{\partial C}{\partial \alpha}, \\
\sum_{i=1}^{n} \operatorname{In}\left(1-w_{i}\right)=-\frac{n}{C} \frac{\partial C}{\partial \beta}, \\
\sum_{i=1}^{n} \frac{w_{i} I_{\nu-1}\left(c w_{i}\right)}{I_{\nu}\left(c w_{i}\right)}=\frac{n \nu}{c}-\frac{n}{C} \frac{\partial C}{\partial c}, \\
\sum_{i=1}^{n} \frac{\partial I_{\nu}\left(c w_{i}\right) / \partial \nu}{I_{\nu}\left(c w_{i}\right)}=-\frac{n}{C} \frac{\partial C}{\partial \nu} .
\end{gathered}
$$


Assuming (2.1) as the prior, the Bayes estimate of the binomial parameter, say $p$, is

$$
\begin{aligned}
E(p \mid x) & \\
= & \frac{C c^{\nu} \Gamma(x+1+\alpha+v) \Gamma(n-x+\beta)}{2^{\nu} \Gamma(n+1+\alpha+\beta+\nu) \Gamma(\nu+1)} \\
& \times{ }_{2} F_{3}\left(\frac{1+x+\alpha+v}{2}, \frac{x+\alpha+v}{2}+1 ; \nu+1, \frac{n+1+\alpha+\beta+v}{2}, \frac{n+\alpha+\beta+v}{2}+1 ; \frac{c^{2}}{4}\right),
\end{aligned}
$$

where $n$ is the number of trials and $x$ is the number of successes.

\section{BB distribution II}

The second generalization of (1.1) is given by the pdf

$$
f(x)=C x^{\alpha-1}(1-x)^{\beta-1} \exp (c x) I_{\nu}(c x)
$$

for $0<x<1, \nu>0, \alpha>0, \beta>0$, and $c \geq 0$, where $C$ denotes the normalizing constant. Application of [12, equation (2.15.4.1)] shows that one can determine $C$ as

$$
\frac{1}{C}=\frac{c^{\nu} \Gamma(\alpha+\nu) \Gamma(\beta)}{2^{\nu} \Gamma(\alpha+\beta+\nu) \Gamma(\nu+1)}{ }_{2} F_{2}\left(\nu+\frac{1}{2}, \alpha+\nu ; 2 \nu+1, \alpha+\beta+\nu ; 2 c\right)
$$

The standard beta pdf (1.1) arises as the particular case of (3.1) for $c=0$ and $\nu=0$. Further particular cases of (3.1) can be obtained using (2.4). The modes of (3.1) are the solutions of

$$
\frac{\alpha-1}{x}-\frac{\beta-1}{x}+\frac{c I_{\nu-1}(c x)}{I_{\nu}(c x)}=\frac{\nu}{c}-c
$$

There could be more than one mode (see Figures 2.1 and 2.2). The $n$th moment of (3.1) can be written as

$$
E\left(X^{n}\right)=C \int_{0}^{1} x^{n+\alpha-1}(1-x)^{\beta-1} \exp (c x) I_{\nu}(c x) d x
$$

and an application of [12, equation (2.15.4.1)] shows that the above reduces to

$$
E\left(X^{n}\right)=\frac{C c^{\nu} \Gamma(n+\alpha+\nu) \Gamma(\beta)}{2^{\nu} \Gamma(n+\alpha+\beta+\nu) \Gamma(\nu+1)}{ }_{2} F_{2}\left(\nu+\frac{1}{2}, n+\alpha+v ; 2 \nu+1, n+\alpha+\beta+v ; 2 c\right)
$$


For a random sample $w_{1}, \ldots, w_{n}$, the MLEs of the four parameters in (3.1) are the solutions of

$$
\begin{gathered}
\sum_{i=1}^{n} \operatorname{In} w_{i}=-\frac{n}{C} \frac{\partial C}{\partial \alpha}, \\
\sum_{i=1}^{n} \operatorname{In}\left(1-w_{i}\right)=-\frac{n}{C} \frac{\partial C}{\partial \beta}, \\
\sum_{i=1}^{n} \frac{w_{i} I_{\nu-1}\left(c w_{i}\right)}{I_{\nu}\left(c w_{i}\right)}+\sum_{i=1}^{n} w_{i}=\frac{n v}{c}-\frac{n}{C} \frac{\partial C}{\partial c}, \\
\sum_{i=1}^{n} \frac{\partial I_{\nu}\left(c w_{i}\right) / \partial \nu}{I_{\nu}\left(c w_{i}\right)}=-\frac{n}{C} \frac{\partial C}{\partial \nu} .
\end{gathered}
$$

Assuming (3.1) as the prior, the Bayes estimate of the binomial parameter, say $p$, is

$$
\begin{aligned}
E(p \mid x)= & \frac{C c^{\nu} \Gamma(1+x+\alpha+\nu) \Gamma(n-x+\beta)}{2^{\nu} \Gamma(n+1+\alpha+\beta+\nu) \Gamma(\nu+1)} \\
& \times{ }_{2} F_{2}\left(\nu+\frac{1}{2}, 1+x+\alpha+v ; 2 \nu+1, n+1+\alpha+\beta+v ; 2 c\right),
\end{aligned}
$$

where $n$ is the number of trials and $x$ is the number of successes.

\section{BB distribution III}

The third and final generalization of (1.1) is given by the pdf

$$
f(x)=C x^{\alpha-1}(1-x)^{\beta-1} \exp (-c x) I_{\nu}(c x)
$$

for $0<x<1, \nu>0, \alpha>0, \beta>0$, and $c \geq 0$, where $C$ denotes the normalizing constant. Application of [12, equation (2.15.4.1)] shows that one can determine $C$ as

$$
\frac{1}{C}=\frac{c^{\nu} \Gamma(\alpha+\nu) \Gamma(\beta)}{2^{\nu} \Gamma(\alpha+\beta+\nu) \Gamma(\nu+1)}{ }_{2} F_{2}\left(\nu+\frac{1}{2}, \alpha+v ; 2 \nu+1, \alpha+\beta+\nu ;-2 c\right) .
$$

The standard beta pdf (1.1) arises as the particular case of (4.1) for $c=0$ and $\nu=0$. Further particular cases of (4.1) can be obtained using (2.4). The modes of (4.1) are the solutions of

$$
\frac{\alpha-1}{x}-\frac{\beta-1}{x}+\frac{c I_{\nu-1}(c x)}{I_{\nu}(c x)}=\frac{\nu}{c}+c .
$$


There could be more than one mode (see Figures 2.1 and 2.2). The $n$th moment of (4.1) can be written as

$$
E\left(X^{n}\right)=C \int_{0}^{1} x^{n+\alpha-1}(1-x)^{\beta-1} \exp (-c x) I_{\nu}(c x) d x
$$

and an application of [12, equation (2.15.4.1)] shows that the above reduces to

$$
E\left(X^{n}\right)=\frac{C c^{\nu} \Gamma(n+\alpha+\nu) \Gamma(\beta)}{2^{\nu} \Gamma(n+\alpha+\beta+\nu) \Gamma(\nu+1)}{ }_{2} F_{2}\left(\nu+\frac{1}{2}, n+\alpha+v ; 2 \nu+1, n+\alpha+\beta+v ;-2 c\right) .
$$

For a random sample $w_{1}, \ldots, w_{n}$, the MLEs of the four parameters in (4.1) are the solutions of

$$
\begin{gathered}
\sum_{i=1}^{n} \operatorname{In} w_{i}=-\frac{n}{C} \frac{\partial C}{\partial \alpha}, \\
\sum_{i=1}^{n} \operatorname{In}\left(1-w_{i}\right)=-\frac{n}{C} \frac{\partial C}{\partial \beta}, \\
\sum_{i=1}^{n} \frac{w_{i} I_{\nu-1}\left(c w_{i}\right)}{I_{\nu}\left(c w_{i}\right)}-\sum_{i=1}^{n} w_{i}=\frac{n \nu}{c}-\frac{n}{C} \frac{\partial C}{\partial c}, \sum_{i=1}^{n} \frac{\partial I_{\nu}\left(c w_{i}\right) / \partial \nu}{I_{\nu}\left(c w_{i}\right)}=-\frac{n}{C} \frac{\partial C}{\partial \nu} .
\end{gathered}
$$

Assuming (4.1) as the prior, the Bayes estimate of the binomial parameter, say $p$, is

$$
\begin{aligned}
E(p \mid x)= & \frac{C c^{\nu} \Gamma(1+x+\alpha+\nu) \Gamma(n-x+\beta)}{2^{\nu} \Gamma(n+1+\alpha+\beta+\nu) \Gamma(\nu+1)} \\
& \times{ }_{2} F_{2}\left(\nu+\frac{1}{2}, 1+x+\alpha+v ; 2 v+1, n+1+\alpha+\beta+v ;-2 c\right),
\end{aligned}
$$

where $n$ is the number of trials and $x$ is the number of successes.

\section{Application}

We now illustrate an application of the proposed beta distributions to consumer price index data. We collected the data on this index for the six countries: United States, United Kingdom, Japan, Canada, Germany, and Australia. The data were extracted from the website http://www.globalfindata.com/ (go to "Sample Data" under "Database" and then look under "Consumer Price Indices" for the closing value of the index) and the range of data for each country is shown in Table 5.1.

Taking the ratio $W=X /(X+Y)$, we attempted to model the relative economic performance of each country against another over the range of overlapping years. This yields 15 data sets for the variable $W$. As expected, some of the data for $W$ appeared to concentrate to a subinterval of $[0,1]$ and so suitable location-scale transformations were applied to make the data span from 0 to 1 . For each data set, we fitted the standard beta distribution given by (1.1) and the BB III distribution given by (4.1) with $\nu$ fixed as $v=1$. The 
Table 5.1. Countries and years of data.

\begin{tabular}{lc}
\hline Country & Range of data \\
\hline Australia & 1901 to 2003 \\
Canada & 1910 to 2003 \\
Germany & 1923 to 2003 \\
Japan & 1868 to 2003 \\
United Kingdom & 1800 to 2003 \\
United States & 1820 to 2003 \\
\hline
\end{tabular}

two distributions were fitted by the method of maximum likelihood. The MLEs of the two parameters in (1.1) are obtained by solving the equations

$$
\begin{gathered}
\sum_{i=1}^{n} \operatorname{In} w_{i}=n \Psi(\alpha)-n \Psi(\alpha+\beta), \\
\sum_{i=1}^{n} \operatorname{In}\left(1-w_{i}\right)=n \Psi(\beta)-n \Psi(\alpha+\beta),
\end{gathered}
$$

where $\Psi(x)=d \operatorname{In} \Gamma(x) / d x$ is the digamma function. The MLEs of $(\alpha, \beta, c)$ in (4.1) with $v$ fixed as $\nu=1$ are obtained by solving (4.6).

The results of the fits were remarkable. In each fit, the maximized log-likelihood for (4.1) turned up significantly higher than that for the standard beta model. Here, we give details for just two of the 15 data sets.

(i) For the (United States, United Kingdom) data set shown in Table A.1 of the appendix the fitted estimates were $\hat{\alpha}=1.392, \hat{\beta}=1.230$ with $\log L=5.145$ for the standard beta model (1.1); and $\hat{\alpha}=0.820, \hat{\beta}=-3.180, \hat{c}=1.571$ with $\log L=$ 7.647 for the BB III model (4.1). The corresponding fitted densities superimposed with the empirical density are shown in Figure 2.1 (the empirical density computed using the hist command in the $R$ software package).

(ii) For the (United States, Germany) data set shown in Table A.2 of the appendix the fitted estimates were $\hat{\alpha}=0.914, \hat{\beta}=1.130$ with $\log L=1.494$ for the standard beta model (1.1); and $\hat{\alpha}=1.405, \hat{\beta}=2.370, \hat{c}=7.828 \times 10^{-6}$ with $\log L=5.198$ for the BB III model (4.1). The corresponding fitted densities superimposed with the empirical density are shown in Figure 2.2 (the empirical density computed using the hist command in the $R$ software package).

So, we can conclude at least in this situation that the beta Bessel models are better than the one based on the standard beta distribution.

\section{Appendix}

Tables A.1 and A.2 provide the data on consumer price indices for the United States and the United Kingdom (years of overlap: 1820-2003) and for the United States and Germany (years of overlap: 1923-2003). 
A. K. Gupta and S. Nadarajah 11

Table A.1. Consumer price index data for the United States and the United Kingdom for the years 1820-2003.

\begin{tabular}{|c|c|c|c|c|c|c|c|c|}
\hline Year & US CPI & UK CPI & Year & US CPI & UK CPI & Year & US CPI & UK CPI \\
\hline 1820 & 6.2 & 4.9 & 1882 & 7.7 & 4.1 & 1944 & 17.8 & 7.4 \\
\hline 1821 & 5.9 & 4.3 & 1883 & 7.5 & 4.3 & 1945 & 18.2 & 7.5 \\
\hline 1822 & 6.1 & 3.7 & 1884 & 7.3 & 3.9 & 1946 & 21.5 & 7.5 \\
\hline 1823 & 5.8 & 4.0 & 1885 & 7.2 & 3.7 & 1947 & 23.4 & 7.8 \\
\hline 1824 & 5.5 & 4.3 & 1886 & 7.2 & 3.4 & 1948 & 24.1 & 8.1 \\
\hline 1825 & 5.6 & 5.0 & 1887 & 7.3 & 3.4 & 1949 & 23.6 & 8.4 \\
\hline 1826 & 5.3 & 4.8 & 1888 & 7.3 & 3.4 & 1950 & 25.0 & 8.6 \\
\hline 1827 & 5.5 & 4.5 & 1889 & 7.3 & 3.4 & 1951 & 26.5 & 9.6 \\
\hline 1828 & 5.5 & 4.3 & 1890 & 7.3 & 3.4 & 1952 & 26.7 & 10.2 \\
\hline 1829 & 5.6 & 4.3 & 1891 & 7.3 & 3.6 & 1953 & 26.9 & 10.4 \\
\hline 1830 & 5.2 & 4.1 & 1892 & 7.3 & 3.6 & 1954 & 26.7 & 10.8 \\
\hline 1831 & 5.4 & 4.5 & 1893 & 7.3 & 3.3 & 1955 & 26.8 & 11.3 \\
\hline 1832 & 5.5 & 4.2 & 1894 & 7.1 & 3.5 & 1956 & 27.6 & 11.7 \\
\hline 1833 & 5.6 & 3.9 & 1895 & 7.0 & 3.5 & 1957 & 28.4 & 12.2 \\
\hline 1834 & 4.9 & 3.6 & 1896 & 7.0 & 3.4 & 1958 & 28.9 & 12.4 \\
\hline 1835 & 5.7 & 3.7 & 1897 & 7.0 & 3.5 & 1959 & 29.4 & 12.4 \\
\hline 1836 & 6.5 & 4.1 & 1898 & 7.1 & 3.5 & 1960 & 29.8 & 12.6 \\
\hline 1837 & 6.9 & 4.2 & 1899 & 7.3 & 3.4 & 1961 & 30.0 & 13.2 \\
\hline 1838 & 6.8 & 4.2 & 1900 & 7.4 & 3.4 & 1962 & 30.4 & 13.5 \\
\hline 1839 & 6.8 & 4.5 & 1901 & 7.6 & 3.3 & 1963 & 30.9 & 13.8 \\
\hline 1840 & 5.7 & 4.6 & 1902 & 7.8 & 3.3 & 1964 & 31.2 & 14.5 \\
\hline 1841 & 5.7 & 4.5 & 1903 & 7.8 & 3.4 & 1965 & 31.8 & 15.1 \\
\hline 1842 & 5.3 & 4.2 & 1904 & 7.9 & 3.4 & 1966 & 32.9 & 15.7 \\
\hline 1843 & 4.9 & 3.7 & 1905 & 8.1 & 3.4 & 1967 & 33.9 & 16.1 \\
\hline 1844 & 5.0 & 3.7 & 1906 & 8.5 & 3.4 & 1968 & 35.5 & 17.0 \\
\hline 1845 & 5.2 & 3.9 & 1907 & 8.8 & 3.5 & 1969 & 37.7 & 17.8 \\
\hline 1846 & 5.6 & 4.0 & 1908 & 8.8 & 3.4 & 1970 & 39.8 & 19.2 \\
\hline 1847 & 5.6 & 4.5 & 1909 & 9.3 & 3.5 & 1971 & 41.1 & 20.9 \\
\hline 1848 & 4.9 & 4.0 & 1910 & 9.3 & 3.5 & 1972 & 42.5 & 22.5 \\
\hline 1849 & 5.2 & 3.7 & 1911 & 9.5 & 3.6 & 1973 & 46.2 & 24.9 \\
\hline
\end{tabular}


Table A.1. Continued.

\begin{tabular}{|c|c|c|c|c|c|c|c|c|}
\hline Year & US CPI & UK CPI & Year & US CPI & UK CPI & Year & US CPI & UK CPI \\
\hline 1850 & 4.9 & 3.5 & 1912 & 9.8 & 3.7 & 1974 & 51.9 & 29.6 \\
\hline 1851 & 5.7 & 3.5 & 1913 & 10.0 & 3.8 & 1975 & 55.5 & 37.0 \\
\hline 1852 & 5.7 & 3.5 & 1914 & 10.1 & 4.1 & 1976 & 58.2 & 42.6 \\
\hline 1853 & 6.1 & 4.1 & 1915 & 10.3 & 5.0 & 1977 & 62.1 & 47.8 \\
\hline 1854 & 6.1 & 4.6 & 1916 & 11.6 & 6.1 & 1978 & 67.7 & 51.8 \\
\hline 1855 & 6.4 & 4.6 & 1917 & 13.7 & 6.8 & 1979 & 76.7 & 60.7 \\
\hline 1856 & 6.5 & 4.5 & 1918 & 16.5 & 8.1 & 1980 & 86.3 & 69.9 \\
\hline 1857 & 6.7 & 4.6 & 1919 & 18.9 & 8.3 & 1981 & 94.0 & 78.3 \\
\hline 1858 & 6.6 & 4.3 & 1920 & 19.4 & 9.8 & 1982 & 97.6 & 82.5 \\
\hline 1859 & 6.0 & 4.4 & 1921 & 17.3 & 7.1 & 1983 & 101.3 & 86.9 \\
\hline 1860 & 5.8 & 4.7 & 1922 & 16.9 & 6.6 & 1984 & 105.3 & 90.9 \\
\hline 1861 & 6.0 & 4.7 & 1923 & 17.3 & 6.5 & 1985 & 109.3 & 96.0 \\
\hline 1862 & 6.6 & 4.6 & 1924 & 17.3 & 6.6 & 1986 & 110.5 & 99.6 \\
\hline 1863 & 7.5 & 4.1 & 1925 & 17.9 & 6.4 & 1987 & 115.4 & 103.3 \\
\hline 1864 & 9.1 & 4.3 & 1926 & 17.7 & 6.4 & 1988 & 120.5 & 110.3 \\
\hline 1865 & 9.8 & 4.5 & 1927 & 17.3 & 6.2 & 1989 & 126.1 & 118.8 \\
\hline 1866 & 9.9 & 4.7 & 1928 & 17.1 & 6.1 & 1990 & 133.8 & 129.9 \\
\hline 1867 & 9.8 & 4.8 & 1929 & 17.2 & 6.1 & 1991 & 137.9 & 135.7 \\
\hline 1868 & 9.4 & 4.6 & 1930 & 16.1 & 5.6 & 1992 & 141.9 & 139.2 \\
\hline 1869 & 9.1 & 4.5 & 1931 & 14.6 & 5.4 & 1993 & 145.8 & 141.9 \\
\hline 1870 & 8.7 & 4.5 & 1932 & 13.1 & 5.2 & 1994 & 149.7 & 146.0 \\
\hline 1871 & 8.5 & 4.8 & 1933 & 13.2 & 5.2 & 1995 & 153.5 & 150.7 \\
\hline 1872 & 8.6 & 5.0 & 1934 & 13.4 & 5.3 & 1996 & 158.6 & 154.4 \\
\hline 1873 & 8.4 & 5.2 & 1935 & 13.8 & 5.4 & 1997 & 161.3 & 160.0 \\
\hline 1874 & 8.4 & 5.1 & 1936 & 14.0 & 5.6 & 1998 & 163.9 & 164.4 \\
\hline 1875 & 8.0 & 4.7 & 1937 & 14.4 & 5.9 & 1999 & 168.3 & 167.3 \\
\hline 1876 & 7.7 & 4.9 & 1938 & 14.0 & 5.7 & 2000 & 174.0 & 172.2 \\
\hline 1877 & 7.3 & 4.8 & 1939 & 14.0 & 6.4 & 2001 & 176.7 & 173.4 \\
\hline 1878 & 6.8 & 4.6 & 1940 & 14.1 & 7.2 & 2002 & 180.9 & 178.5 \\
\hline 1879 & 7.3 & 4.4 & 1941 & 15.5 & 7.4 & 2003 & 184.3 & 183.5 \\
\hline 1880 & 7.5 & 4.2 & 1942 & 16.9 & 7.3 & - & - & - \\
\hline 1881 & 7.9 & 4.4 & 1943 & 17.4 & 7.3 & - & - & - \\
\hline
\end{tabular}


Table A.2. Consumer price index data for the United States and Germany for the years 1923-2003.

\begin{tabular}{l|cl|l|ll|l|ll}
\hline Year & US CPI & DE CPI & Year & US CPI & DE CPI & Year & US CPI & DE CPI \\
\hline 1923 & 17.3 & 17.8326 & 1950 & 25 & 22.8529 & 1977 & 62.1 & 55.7936 \\
1924 & 17.3 & 17.2442 & 1951 & 26.5 & 25.7652 & 1978 & 67.7 & 57.1635 \\
1925 & 17.9 & 17.9829 & 1952 & 26.7 & 26.9921 & 1979 & 76.7 & 60.2769 \\
1926 & 17.7 & 18.3777 & 1953 & 26.9 & 26.2559 & 1980 & 86.3 & 63.5772 \\
1927 & 17.3 & 19.2692 & 1954 & 26.7 & 26.9921 & 1981 & 94 & 67.8739 \\
1928 & 17.1 & 19.4475 & 1955 & 26.8 & 27.3546 & 1982 & 97.6 & 70.9873 \\
1929 & 17.2 & 19.4348 & 1956 & 27.6 & 27.8025 & 1983 & 101.3 & 72.8554 \\
1930 & 16.1 & 18.0339 & 1957 & 28.4 & 28.3949 & 1984 & 105.3 & 74.2876 \\
1931 & 14.6 & 16.6584 & 1958 & 28.9 & 28.7063 & 1985 & 109.3 & 75.5953 \\
1932 & 13.1 & 15.0537 & 1959 & 29.4 & 29.3289 & 1986 & 110.5 & 74.7858 \\
1933 & 13.2 & 15.3593 & 1960 & 29.8 & 29.5781 & 1987 & 115.4 & 75.533 \\
1934 & 13.4 & 15.5631 & 1961 & 30 & 30.3875 & 1988 & 120.5 & 76.7784 \\
1935 & 13.8 & 15.7159 & 1962 & 30.4 & 31.2593 & 1989 & 126.1 & 79.2839 \\
1936 & 14 & 15.8306 & 1963 & 30.9 & 32.3179 & 1990 & 133.8 & 81.4561 \\
1937 & 14.4 & 15.8894 & 1964 & 31.2 & 33.0029 & 1991 & 137.9 & 84.1 \\
1938 & 14 & 15.9679 & 1965 & 31.8 & 34.3105 & 1992 & 141.9 & 86.9 \\
1939 & 14 & 16.1052 & 1966 & 32.9 & 35.3069 & 1993 & 145.8 & 90.6 \\
1940 & 14.1 & 16.6545 & 1967 & 33.9 & 35.4936 & 1994 & 149.7 & 92.9 \\
1941 & 15.5 & 16.9487 & 1968 & 35.5 & 36.3032 & 1995 & 153.5 & 94.3 \\
1942 & 16.9 & 17.3018 & 1969 & 37.7 & 37.0504 & 1996 & 158.6 & 95.7 \\
1943 & 17.4 & 17.6353 & 1970 & 39.8 & 38.5448 & 1997 & 161.3 & 97.6 \\
1944 & 17.8 & 17.9688 & 1971 & 41.1 & 40.662 & 1998 & 163.9 & 98 \\
1945 & 18.2 & 18.8093 & 1972 & 42.5 & 43.2774 & 1999 & 168.3 & 99.1 \\
1946 & 21.5 & 20.745 & 1973 & 46.2 & 46.6399 & 2000 & 174 & 101.2 \\
1947 & 23.4 & 21.318 & 1974 & 51.9 & 49.3175 & 2001 & 176.7 & 102.8 \\
1948 & 24.1 & 25.4258 & 1975 & 55.5 & 51.995 & 2002 & 180.9 & 104 \\
1949 & 23.6 & 23.6096 & 1976 & 58.2 & 53.9254 & 2003 & 184.3 & 105.1 \\
\hline & & & & & & & &
\end{tabular}

\section{Acknowledgments}

The authors would like to thank the Editor-in-Chief and the two referees for carefully reading the paper and for their great help in improving the paper.

\section{References}

[1] C. Armero and M. J. Bayarri, Prior assessments for prediction in queues, The Statistician 43 (1994), $139-153$.

[2] R. E. Barlow and F. Proschan, Statistical Theory of Reliability and Life Testing: Probability Models, Holt, Rinehart and Winston, New York, 1975.

[3] M. B. Gordy, Computationally convenient distributional assumptions for common-value auctions, Computational Economics 12 (1998), no. 1, 61-78. 
[4] I. S. Gradshteyn and I. M. Ryzhik, Table of Integrals, Series, and Products, 6th ed., Academic Press, California, 2000.

[5] A. K. Gupta and S. Nadarajah, Handbook of Beta Distribution and Its Applications, Statistics: Textbooks and Monographs, vol. 174, Marcel Dekker, New York, 2004.

[6] N. L. Johnson, S. Kotz, and N. Balakrishnan, Continuous Univariate Distributions. Vol. 2, 2nd ed., Wiley Series in Probability and Mathematical Statistics: Applied Probability and Statistics, John Wiley \& Sons, New York, 1995.

[7] Z. A. Karian and E. J. Dudewicz, Fitting Statistical Distributions. The Generalized Lambda Distribution and Generalized Bootstrap Methods, CRC Press, Florida, 2000.

[8] D. L. Libby and M. R. Novick, Multivariate generalized beta-distributions with applications to utility assessment, Journal of Educational Statistics 7 (1982), no. 4, 271-294.

[9] J. B. McDonald and D. O. Richards, Model selection: some generalized distributions, Communications in Statistics. Theory and Methods 16 (1987), no. 4, 1049-1074.

[10] J. B. McDonald and Y. J. Xu, A generalization of the beta distribution with applications, Journal of Econometrics 66 (1995), no. 1-2, 133-152.

[11] A. P. Prudnikov, Yu. A. Brychkov, and O. I. Marichev, Integrals and Series. Vol. 1. Elementary Functions, Gordon \& Breach Science, New York, 1986.

[12] _ Integrals and Series. Vol. 2. Special Functions, Gordon \& Breach Science, New York, 1986.

[13] D. O. Richards and J. B. McDonald, A general methodology for determining distributional forms with applications in reliability, Journal of Statistical Planning and Inference 16 (1987), no. 3, $365-376$.

Arjun K. Gupta: Department of Mathematics and Statistics, Bowling Green State University, Bowling Green, OH 43403, USA

E-mail address: gupta@bgsu.edu

Saralees Nadarajah: School of Mathematics, University of Manchester, Oxford Road, Manchester M13 9PL, UK

E-mail address: snadaraj@unlserve.unl.edu 


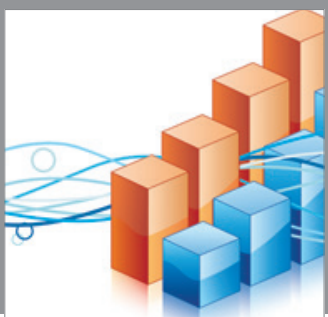

Advances in

Operations Research

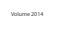

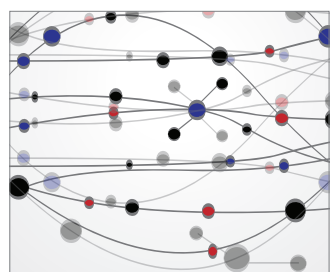

\section{The Scientific} World Journal
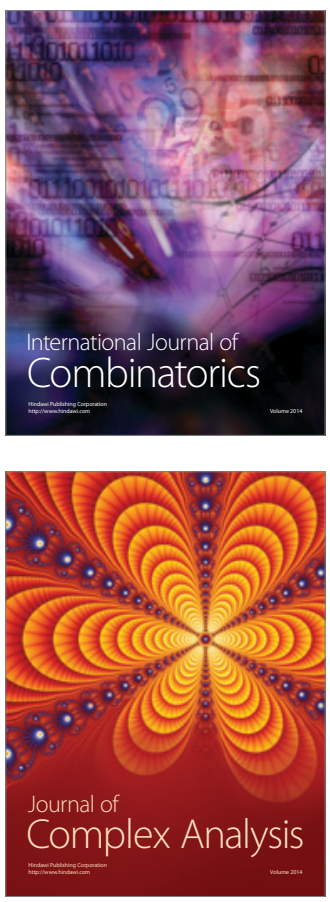

International Journal of

Mathematics and

Mathematical

Sciences
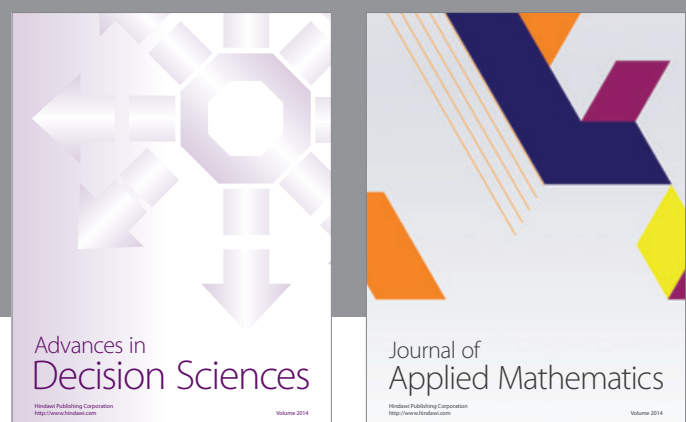

Journal of

Applied Mathematics
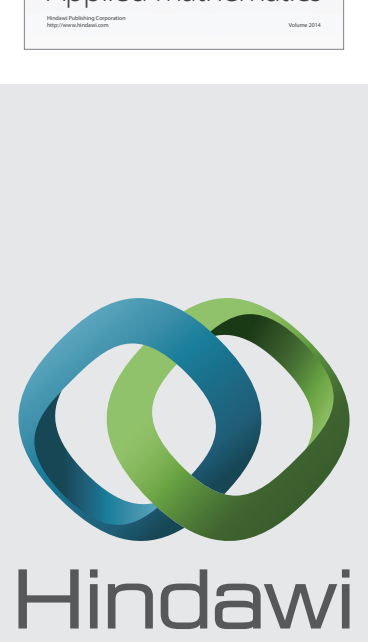

Submit your manuscripts at http://www.hindawi.com
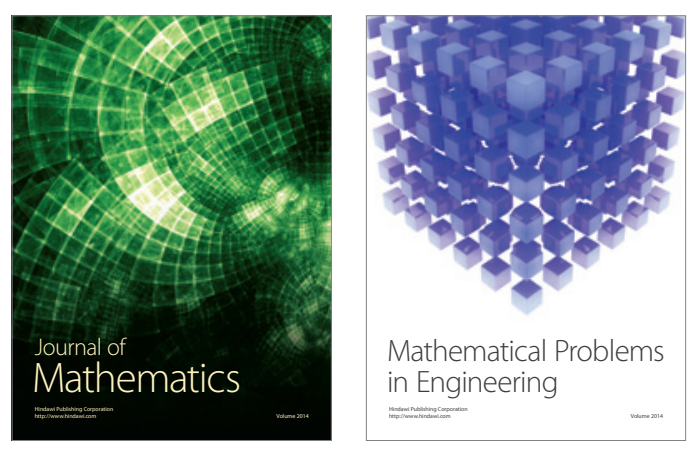

Mathematical Problems in Engineering
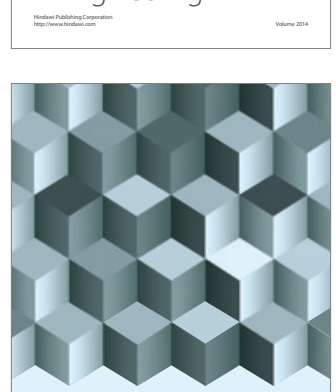

Journal of

Function Spaces
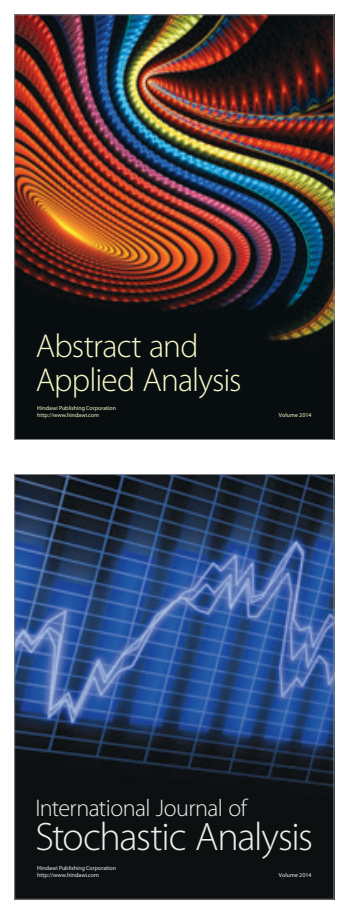

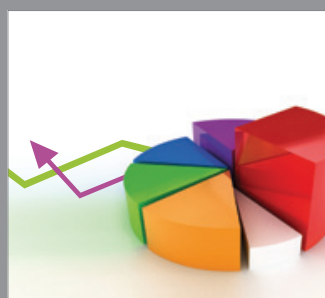

ournal of

Probability and Statistics

Promensencen
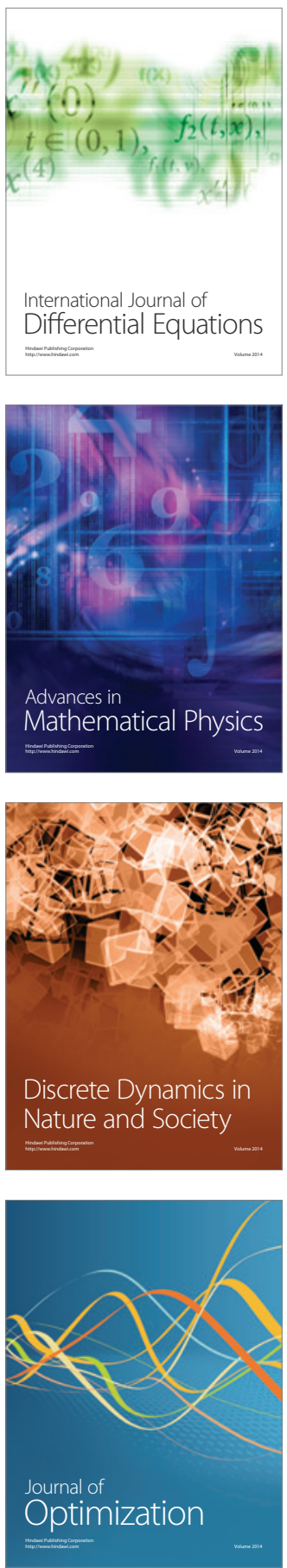